\title{
Reflets
}

Revue ontaroise d'intervention sociale et communautaire

\section{Langue minoritaire et services de santé mentale en l'an 2000 : droits et besoins des francophones de Toronto}

\section{Françoise Boudreau}

Volume 5, numéro 2, automne 1999

La santé des francophones de l’Ontario

URI : https://id.erudit.org/iderudit/026273ar

DOI : https://doi.org/10.7202/026273ar

Aller au sommaire du numéro

Éditeur(s)

Reflets : Revue ontaroise d'intervention sociale et communautaire

ISSN

1203-4576 (imprimé)

1712-8498 (numérique)

Découvrir la revue

Citer cet article

Boudreau, F. (1999). Langue minoritaire et services de santé mentale en l'an 2000 : droits et besoins des francophones de Toronto. Reflets, 5(2), 123-154.

https://doi.org/10.7202/026273ar

Tous droits réservés (C) Reflets : Revue ontaroise d'intervention sociale et communautaire, 1999
Ce document est protégé par la loi sur le droit d'auteur. L'utilisation des services d'Érudit (y compris la reproduction) est assujettie à sa politique d'utilisation que vous pouvez consulter en ligne.

https://apropos.erudit.org/fr/usagers/politique-dutilisation/ 


\title{
Langue minoritaire et services de santé mentale en l'an 2000 : droits et besoins des francophones de Toronto ${ }^{1}$
}

\author{
F rançoise B oudreau
}

\section{Introduction}

D ans le cadre de la mise en application de la $R$ éforme ontarienne en santé mentale qui promettait de «M ettre la personne au premier plan » (1993), le Conseil régional de santé du Toronto métropolitain ${ }^{2}$ a créé un comité chargé d'effectuer une étude de besoins auprès des francophones de la région souffrant de problèmes sévères et persistants de santé mentale $e^{3}$. C elle-ci s'avérait essentielle en vertu de la $\mathrm{L}$ oi 8 et de plus, des documents de la réforme classifiaient les francophones de la province comme étant un des groupes qui n'était pas « servis adéquatement » dans ce domaine (1993: 31)4. Ici, notre but n'est pas de présenter un compte- rendu du rapport (Boudreau, 1996), mais d'en revoir certaines données tant quantitatives que qualitatives afin de présenter un portrait inusité d'une population de francophones vivant avec de graves problèmes de santé mentale dansla région deToronto.

Le présent article aura deux composantes majeures. D ans un premier temps, à l'aide des données de StatistiquesC anada, nous avons tenté d'estimer l'ampleur de la demande potentielle 
des francophones à Toronto pour des services psychiatriques. Q uelle est l'étendue des besoins? D oit-on parler de dizaines, de centaines ou même de milliers de personnes qui, dans la région deToronto souffriraient de problèmes de santé mentale et auraient droit aux services en français? Pour faire cette exploration statistique, nous avons fait appel au Supplément sur la santé mentale de l'E nquête sur la Santé de l'O ntario de 1991 et à l'ai de des variables disponibles, nous avons comparé, chez les francophones et les non francophones, l'ampleur des besoins exprimés et le taux d'utilisation des services. Les membres de la minorité francophone auraient-ils des niveaux de besoins et des comportements d'utilisation des services de santé mentale différents " des autres » 0 ntariens? Si oui, quels indices le Supplément peut-il nous offrir en vue d'une explication? L'expérience du "minoritaire », telle que décrite par les littéraires et sociologues de cette francophonie, peut-elle légitimement faire partie de cette explication? Q uelques réflexions sur le portrait épidémiologique ainsi produit serviront de porte d'entrée vers la composante qualitative de cette étude où nous mettrons, effectivement, « la personne au premier plan ». À cette fin, nous avons interviewé 22 francophones aux prises avec des problèmes de santé mentale dans la région deToronto et qui ont été ou sont toujours des utilisateurs de services, soit en anglais, soit en français. En leur donnant la parole, nous souhaitions surtout entendre leurs points de vue concernant la nécessité d'avoir des services en

« N ous désirions ainsi avoir un aperçu des « trajectoires suivies», des barrières auxquelles ils se sont heurtés des désirs et des laaunes à combler. » français. N ous désirions ainsi avoir un aperçu des «trajectoires suivies ", des barrières auxquelles ils se sont heurtés, des désirs et des lacunes à combler. Sans généraliser, ni prétendre à I'exhaustivité des expériences, ces témoignages nous ont permis de saisir le tragique de la situation de ces personnes et de personnes comme elles, à l'aube de l'an 2000.

\section{Bref portrait épidémiologique}

Toute réponse à la question concernant le nombre de francophones àToronto et de là, de la grosseur du bassin de population ayant droit à des services de santé mentale en français, dépend de 
la définition choisie pour y répondre. L'accent peut être mis sur les 2245 personnes identifiées par Statistiques Canada qui, en 1991, ne parlent que le français comme langue officielle àToronto ou encore, sur les 11110 qui ne parlent que le françaisà la maison ou les 40570 qui déclarent avoir le français comme langue maternelle ou même, sur le groupe très disparate des 139035 personnes qui disent connaitre le français et l'anglais comme langues officielles, mais dont bons nombres ne songeraient jamais à se prévaloir des droits conférés par la L oi 8 . U ne variable linguistique créée par Statistique $C$ anada justement pour les fins d'application de la $L$ oi sur les langues officelles et selon laquelle «la minorité francophone » deToronto compterait, en 1991, 42068 personnes nous offre un bon compromis (Tableau 1$)^{5}$.

\section{Tableau 1 - Population selon la première langue parlée et «minorité francophone ». O ntario, Toronto et Région du Grand Toronto, 1991}

\begin{tabular}{|l|r|r|r|}
\hline $\begin{array}{l}\text { Première langue } \\
\text { officielle parlée }\end{array}$ & \multicolumn{1}{|c|}{ Ontario } & \multicolumn{1}{|c|}{ Toronto } & $\begin{array}{l}\text { Région du Grand } \\
\text { Toronto }\end{array}$ \\
\hline Total & 9977055 & 2255170 & 4282195 \\
\hline Anglais & 9255865 & 2094120 & 4052640 \\
\hline Français & 483445 & 31610 & 60815 \\
\hline Anglais et français & 52410 & 20915 & 29255 \\
\hline $\begin{array}{l}\text { Ni anglais, ni } \\
\text { français }\end{array}$ & 185335 & 108525 & 139450 \\
\hline $\begin{array}{l}\text { Minorité } \\
\text { francophone }\end{array}$ & 509650 & 42068 & 75443 \\
\hline
\end{tabular}

Source: Statistique $C$ anada. R ecensement de 1991 (échantillon de $20 \%$ ). Totalisations spédales produites par Statistiques $\mathrm{C}$ anada pour $\mathrm{R}$ ené $\mathrm{G}$ uindon, $\mathrm{AC} O \mathrm{R} \mathrm{D}$.

Bien que chacune de ces personnes soit en droit de recevoir des services en français, nous n'avons pas d'informations factuelles concernant le nombre exact de personnes qui auraient, à tout moment, besoin de ces services ou le nombre exact de personnes qui s'en prévalent dans quelle que langue que ce soit. $\mathrm{N}$ ous ne 
«... le taux annuel de prévalence de toutes formes de troubles mentaux graves, modérés ou plus légers, calculé au cours de l'année précédant l'enquête, se situait à $19,5 \%$ pour tous les 0 ntariens » pouvons que faire certaines estimations. Ainsi, le ministère de la Santé de l'0 ntario situe à $2 \%$, le taux standard de prévalence de maladies mentales de nature " grave, sévère et persistante » ou "chronique »chez les 0 ntariens (O ntario H ealth Survey 1990: 1). U ne simple extrapolation suggère donc que dans son ensemble, la minorité francophone de Toronto et celle plus englobante du Grand Toronto compteraient respectivement un total de 841 et de 1509 personnesqui souffrent de problèmes sévères et persistants en santé mentale. Parmi celles-ci, il y aurait 45 personnesàToronto et 72 dans le grand Toronto qui, de surcroît, ne savent pas du tout parler anglais.

Les résultats du Supplément sur la santé mentale de l'E nquête Santé de l'0 ntario de 1991 jettent un peu plus de lumière sur la situation ${ }^{7}$. Selon eux, le taux annuel de prévalence de toutes formes de troubles mentaux graves, modérés ou plus légers, calculé au cours de l'année précédant l'enquête, se situait à $19,5 \%$ pour tous les 0 ntariens. Selon ces résultats, en un an, près d'une personne sur cing en O ntario aurait vécu au moins un des troubles de santé mentale mesurés par l'enquête. C e ratio de un sur cinq est déjà bien connu.

$\mathrm{N}$ ous nous sommes alors demandé si le taux de prévalence, de même que le taux annuel d'utilisation de services révélé par l'enquête, car toutes les personnes affectées ne consultent pas nécessairement, serait semblable pour les francophones et les non-francophones de la province ${ }^{8}$. D éjà, $N$ éré St-Amand avait démontré que les A cadiens du N ouveau-B runswick étaient «surreprésentés de façon significative, au niveau des traitements en psychiatrie » (1985: 164). À son tour, Snow (1987: 85) rapporte que «dansle nord du N ouveau-B runswick, où vivent la majorité des Acadiennes et Acadiens, on peut observer un taux d'internement en psychiatrie double de celui de la moyenne canadienne » (cité dansSt-A mand etVuong 1994: 182). Par contre, Woolfson (1975) et Langelier (1982) ont établi que les FrancoA méricains sous-utilisaient les services de santé mentale. Certaines études attestent d'un niveau de prévalence élevé ou plus élevé de troubles de nature mentale chez les groupes minoritaires (Fisher 1969; N eighbors 1986; Leong 1986; 
Passamanick 1963; Wallen et al. 1992), accompagné d'un taux plus bas d'utilisation des services (Woodward et al. 1992), en particulier des services externes (Wallen 1992; Scheffler et M iller, 1989). D'autres études sur le sujet rapportent un taux d'utilisation de l'urgence (Scheffler et M iller 1989; Snow den et Cheung 1990) et un taux de réhospitalisation plus élevés chez les groupes minoritaires (H avassy et $\mathrm{H}$ opkin 1989). D'autres études notent une plus forte proportion d'admissions involontaires (Lindsey et Paul 1989; St-A mand 1985). À leur tour, Bavington et $M$ ajid soulignent que les groupes minoritaires de la Grande-B retagne sous- utilisent les bénéfices du Service N ational de la Santé (I986: 91). Par contre, au $C$ anada, une analyse des premières admissions pour patients schizophrènes a révélé que les groupes culturels et linguistiques minoritaires étaient sur-représentés (Bland et O rn 1981, repris dans Beiser 1988:14). Et pourtant, Woodward démontre que la population américaine d'origine hispanique a un taux de prévalence de troubles mentaux similaire à celui de la population générale, mais un taux d'utilisation des services beaucoup plus faible (Woodward et al. 1992: 224) .

«N os propres calculs, venant du Supplément sur la Santé mentale, révèlent que, chez les francophones de I'O ntario, le taux de prévalence de diagnostics psychiatriques au cours de l'année précédant I'enquête s'élevait à $22,4 \%$ et à $18,1 \%$ chez les autres. »
N os propres calculs, venant du Supplément sur la Santémental $e^{10}$, révèlent que, chez les francophones de l'O ntario, le taux de prévalence de diagnostics psychiatriques au cours de l'année précédant l'enquête s'élevait à $22,4 \%{ }^{11}$ et à $18,1 \%$ chez les autres. D e plus, cette différence demeurait, qu'il s'agisse de francophones du milieu rural comme du milieu urbain, des hommes comme des femmes, des personnes vivant dans le $\mathrm{N}$ ord ontarien comme dans le Sud et C entre Sud (Boudreau, Lin et Barnes 1997). Ainsi, les mêmes taux s'appliqueraient à Timmins et à Toronto. Par extrapolation, nous pouvons donc soutenir qu'en un an, 9423 personnes faisant partie de la minorité francophone de Toronto et 16899 du Grand Toronto auraient besoin de services de santé mentale (Tableau 2). II en serait ainsi pour 503 francophones de Toronto et 810 du Grand Toronto qui ne savent pas du tout s'exprimer en anglais. 
Tableau 2 - Prévalence de problèmes graves et persistants et prévalence de tout problème de santé mentale au cours des douze derniers mois chez les francophones de l'Ontario, de Toronto et du Grand Toronto, selon qu'ils ne parlent que le français comme langue officielle ou qu'ils font partie de la «minorité francophone », 1991

\begin{tabular}{|l|r|r|r|}
\hline $\begin{array}{l}\text { Minorité } \\
\text { francophone }\end{array}$ & Population & $\begin{array}{c}\text { Prévalence de } \\
\text { problèmes graves } \\
\text { et persistants (2\% } \\
\text { de la population) }\end{array}$ & $\begin{array}{c}\text { Prévalence de } \\
\text { tous les problème } \\
\text { en douze mois } \\
\text { (22,4\% de la } \\
\text { population) }\end{array}$ \\
\hline Ontario & 509650 & 10193 & 114161 \\
\hline Grand Toronto & 75443 & 1509 & 16899 \\
\hline Toronto & 42068 & 841 & 9423 \\
\hline $\begin{array}{l}\text { Français seule } \\
\text { langue officielle }\end{array}$ & & & \\
\hline Ontario & 54245 & 1085 & 12151 \\
\hline Grand Toronto & 3615 & 72 & 810 \\
\hline Toronto & 2245 & 45 & 503 \\
\hline
\end{tabular}

Source: E xtrapolations basées sur les données de Statistiques $C$ anada, 1991 et de R ené G uindon, AC O R D, 1994

L eSupplément sur la Santé $M$ entale de l'O ntario recelait autre chose. Q u'il s'agisse de l'année précédant l'enquête ou durant leur vie entière, les francophones éprouvent plus souvent que les nonfrancophones des difficultés au niveau de la santé mentale. C eci apparaît tant au niveau du diagnostic ${ }^{12}$ (voir Figure 1 pour l'année précédant l'enquête) qu'au niveau de la perception que les répondants se font eux-mêmes de leur état de santé mentale (Boudreau 1996).Toutefois, comme le démontre la figure 1, leur taux d'utilisation de ces services ne diffère pas de celui des nonfrancophones (7,5\% et $7,7 \%$ respectivement ). 0 n peut en conclure qu'il semblerait y avoir un niveau de besoins « non comblés », peut-être est-ce beaucoup dire, plus élevé chez les francophones. N on seulement nous ne savons pas dans quelle 
langue les services ont été reçus, mais si ceux-ci ont été comblés à court ou à long terme et avec "satisfaction ». La composante qualitative de cette étude en révélera plus long à ce sujet. $A u$ préalable, cependant, il importe d'explorer plus profondément les données du Supplément afin de voir si celles-ci n'auraient pas quelques indices à offrir pour aider à répondre à deux questions inéluctables: pourquoi les francophones de l'O ntario auraient-ils plus de difficultés et donc, un plus grand besoin de services de santé mentale? Et, pourquoi y aurait-il un niveau plus élevé de besoins dits «non comblés »?

\section{Figure 1 - Besoins de services en santé mentale non comblés chez les francophones et non-francophones de l'O ntario (15-64 ans)}

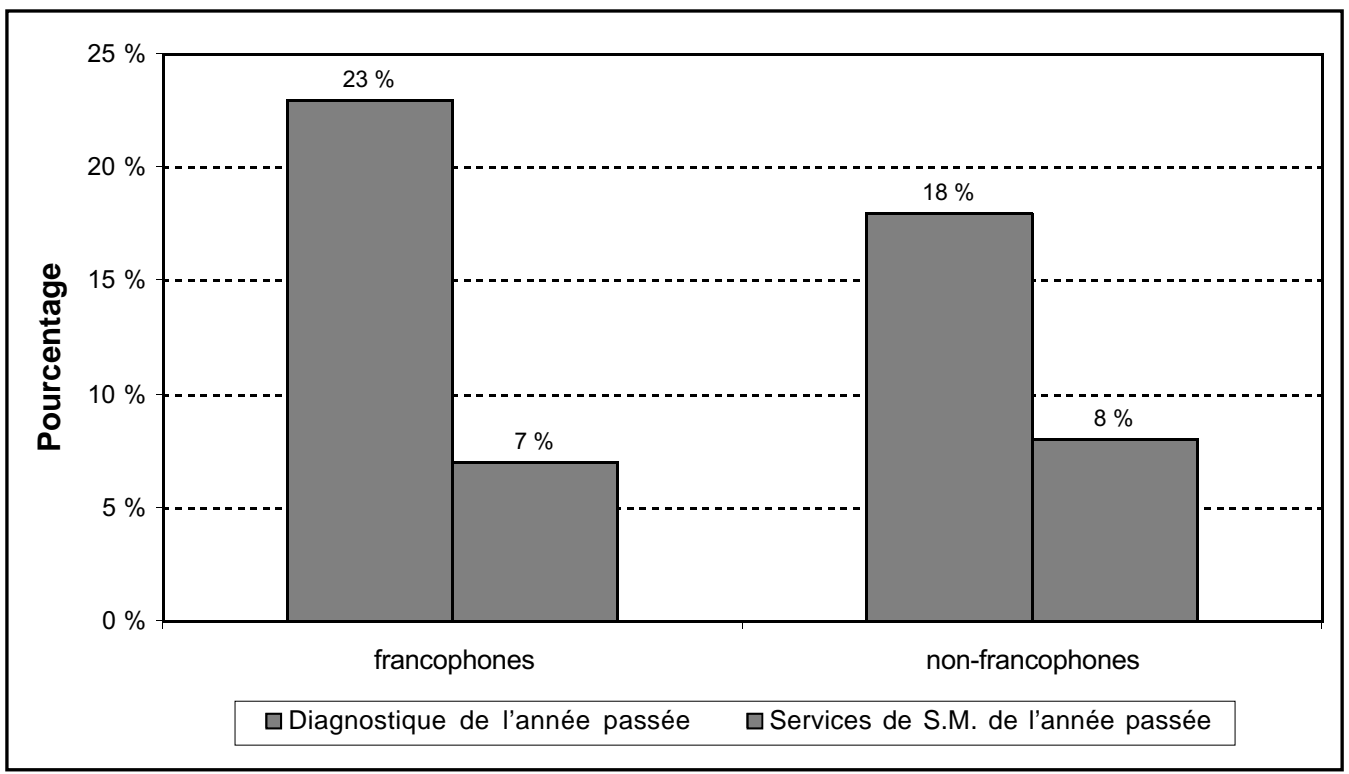

Source : Supplément sur la santé mentale de l'E nquête Santé de l'0 ntario (1991) 


\section{À la recherche d'une explication socio-démographique, culturelle ou linguistique}

«... l'échantillon

francophone du

Supplément sur la Santé mentale reflétait dans toutes ses caractéristiques le profil sociodémographique des francophones de l'0 ntario. »

«... il semble que les répondants francophones seraient un peu plus disposés à admettre qu'ils ont des problèmes de santé mentale $(12,1 \%$ contre $9,8 \%$ ) »
À l'aide des variables disponibles dans le Supplément, nous avons pu explorer, de façon rudimentaire, trois avenues d'explications possibles. S'agit-il de différences essentiellement sociodémographiques? D oit- on parler de particularités attitudinales et culturelles? D oit- on y voir l'effet pénible de barrières linguistiques?

En premier lieu, les différences observées se maintiennent autant chez les hommes ou les femmes, chez les personnes de milieux rural ou urbain, chez celles du N ord de l'O ntario ou du Sud et par conséquent, il semblerait que ce qui affecte ces Francophones les affecte tous également. De plus, l'échantillon francophone du Supplément sur la Santé mentale reflétait dans toutes ses caractéristiques le profil socio-démographique des francophones de l'O ntario. Ilsavaient un niveau d'éducation moins élevé, un revenu familial inférieur, un revenu personnel inférieur, peu importe la région, chez les personnes âgées de 44 ans et plus et surtout, chez les personnes âgées de 55 à 64 ans et enfin, une plus grande proportion de personnes séparées, divorcées ou veuves que les non-francophones. $N$ ous savons de plus que les francophones de l'O ntario présentent un niveau élevé d'analphabétisme (Wagner 1990) et une plus grande mobilité géographique (ACFO 1989). Toutes ces caractéristiques sont, de longue date, reconnues comme des facteurs de risque dans le domaine de la santé mentale (O ntario H ealth Survey 1990).

Au niveau des attitudes soutenues par les francophones face à la santé mentale, les résultats de l'enquête concordent avec certaines tendances à expliquer. En effet, il semble que les répondants francophones seraient un peu plus disposés à admettre qu'ils ont des problèmes de santé mentale $(12,1 \% \text { contre } 9,8 \%)^{13}$. En revanche, ils seraient moins disposés à dire qu'ils se prévaudraient de servicess'ilsavaient un problème grave ( $11,7 \%$ contre $15,4 \%)$. Par ailleurs, moins de francophones que de non-francophones seraient embarrassés d'admettre à leurs amis qu'ils reçoivent des 
services psychiatriques (34,6 \% contre 43,1\%). Par contre, ils se disent tout aussi inconfortables à l'idée de discuter de leurs problèmes personnels avec des professionnels $(20,6 \%$ contre $19,6 \%$ ). D ésir profond de solutionner ses problèmes tout seul, en famille ou entre amis, peur de déranger ou d'attirer l'attention "des autres » et surtout de mal paraitre aux yeux des «étrangers», particulièrement si ces derniers font partie de la majorité anglophone? L'énorme pouvoir de ces sentiments avait été observé en Acadie par St-Amand (1992) et associé à la condition de minoritaire. $D$ ans son étude de la famille canadienne-française traditionnelle au Vermont, Woolfson explique la sous-utilisation des services de santé mentale chez ceux-ci dansces mots: « recourse to outside aid is an indication that the family cannot handle its own affairs. It is a defeat only accepted as a last resort »(1975: 13). Selon Langelier, qui se penche aussi sur la réali ité franco-américaine, cette barrière culturelle serait exacerbée par l'expérience religieuse du confessionnal: «which makes people reluctant to speak out face to face, in daylight, in an open space, to a person not bound by the same religious rules as is the priest »(1982:239). Finalement, une étude plus récente de St-Amand et Vuong auprès d'expsychiatrisés du N ouveau-Brunswick «démontre que le réseau sur lequel s'appuient les ex-psychiatrisés francophonesinterrogés est davantage social (famille, groupes d'entraide), alors que pour la population anglophone, l'appareil institutionnel (psychiatres, institutions et cliniques) jouent un rôle déterminant »(1994: 194). Tout ceci concorde avec les résultats du Supplément selon lesquels les francophones disaient être à la fois moins prêts à discuter de leurs problèmes personnels avec des professionnels et moins

"Q ue les francophones dépendent plus de leurs réseaux d'entraide pourrait expliquer jusqu'à un certain point, une sous-utilisation des services professionnels et institutionnels » embarrassés d'en discuter avec leurs amis. Q ue les francophones dépendent plus de leurs réseaux d'entraide pourrait expliquer jusqu'à un certain point, une sous-utilisation des services professionnels et institutionnels. Est-ce dire que nous trouverons chez les 22 répondants interviewés et qui ont eu recours aux services publics, que les aidants naturels jouent un faible rôle dans leur vie? Ajoutons que cette tendance à recourir à son milieu d'aide naturel pourrait se révéler bénéfique. $N$ éanmoins, il demeure que ces mêmes francophones ont, selon la L oi 8, droit à des services dont ils ne se prévalent pas autant que les autres et 
«En effet, nos constatations semblent offrir encore plus de crédibilité aux condusions de Berry et al. (1987) qui identifient comme facteur de risque en santé mentale, un niveau croissant d'assimilation à une culture majoritaire.» que ceci peut-être aussi dû à une barrière linguistique, socioculturelle ou autre qui ne devrait pas exister.

À ce niveau, la variable «langue parlée à la maison » de l'enquête s'avère fort intéressante et permet de vérifier I'hypothèse suivante. S'il s'agissait d'une barrière principalement linguistique, nous pourrions nous attendre à ce que le taux d'utilisation des services soit plus élevé chez les francophones qui parlent l'anglais à la maison. En effet, ne se sentiraient-ils pas plusà l'aise de parler avec un professionnel, dans la langue utilisée de toutes façons, dans les relations familiales et intimes à la maison?

L'hypothèse n'a pas été confirmée. II n'y avait aucune différence au niveau de l'utilisation des services au cours de l'année précédant l'enquête entre francophones parlant français et francophones parlant anglais à la maison. Q ui plus est, moins de francophones parlant anglais à la maison déclaraient avoir utilisé de tels services au cours de leur vie $(20,8 \%$ contre $24,7 \%)$. Pourtant, une plus forte proportion de ces francophones avaient vécu au moins un problème psychiatrique ( $24,9 \%$ contre $18,6 \%$ ) au cours de l'année précédant l'enquête et ont révélé qu'ils considéraient comme étant «mauvais » leur état de santé mentale ( $24,9 \%$ contre $20,1 \%$ ), soit un sur quatre au lieu de un sur cinq. Les données fort limitées du Supplément ne permettent pas de pousser plus loin notre questionnement, mais conduisent à faire certaines réflexions. $E n$ effet, nos constatations semblent offrir encore plus de crédibilité aux conclusions de Berry et al. (1987) qui identifient comme facteur de risque en santé mentale, un niveau croissant d'assimilation à une culture majoritaire. En clinique, le psychanalyste français Demangeat identifie «l'autre langue comme symptôme » en en parlant comme «la nostalgie d'une langue autre » ou encore, «d'une intimité révolue » avec soi-même ainsi que «la division du sujet »qui emploie la «bilangue »(1989: 373-375) dans la vie de tous les jours.

Est-il aussi légitime de chercher quelqu'explication dans les écrits de nos littérai resfrancophones qui ont constamment utilisé la métaphore de la folie pour illustrer les conséquences de la condition de minoritaire en 0 ntario? N ous pensons, entre autres, aux écrits de Patrice D esbiens qui dédie sa poésie «à ceux qui 
ont faim et sont mangés » (1977). II écrit: «il me manque des morceaux, il en traîne partout » (1978: 68), « je suis plein de trous» (1978:69) ou encore, «Je suis le franco-ontarien dans le Woolworth abandonné de ses rêves» $(1977: 38)^{14}$. C es énoncés illustrent si bien le terrible sentiment "d'aliénation et de dépossession » qui les habitent. $D$ ans un livre au titre bilingue fort révélateur, L'homme invisible/ T he Invisible $M$ an, D esbiens divulgue de façon troublante les sentiments de division et d'invisibilité qui torturent son héros et de là, la culture francoontarienne. Ici, « l'homme invisible n'ose plus rien dire, n'ose plus rien faire » (Desbiens 1981 :33). Si l'on admet que cette «critique acerbe de la société (... ) témoigne spécifiquement de la condition franco-ontarienne » (C arrière 1993 : 308), il ne s'agirait plus d'examiner la plus grande prévalence de troubles mentaux et la sous-utilisation des services uniquement en termes de barrières linguistiques ou attitudinales, mais de faire hommage à l'expérience même du minoritaire décrite par Fernand Dorais comme cet être «fatalement condamné à une incessante dérive. Il est humilié, bafoué, vidé de son appartenance au sens, marginalisé, réduit au silence » (cité dans Parél994:27). Q ui plus est, I'O ntario français serait un "peuple de névrosés», "spectateur de sa propre existence », «infantilisé », «coupé de la véritable intervention dans le monde qui l'entoure et le détermine » (cité dans Paré 1994 : 28). A ux dires de François Paré, l'argumentation est « violente » (1994 : 28) et selon nous, son application à toute une collectivité de termes associés aux symptômes de la folie est dévastatrice. Pourtant, Paré insiste : " la menace de la folie vraie, de la folie démente et destructrice de l'identité, se situe au cœur même des cultures de l'exiguïté et de tous les discours marginaux. C ette folie n'est pas bonne à voir ni à entendre; elle est souffrante, condamne au silence »(1992: 120). Est-ce bien ce que le Supplément sur la santé mentale a mesuré? Est-ce de cette folie que nous observons les manifestations épidémiologiques? La prudence sociologique nous commande de sérieuses réserves!

Bien que ces écrivains de la littérature francophone de I'O ntario se veulent les portes-paroles de leur communauté «touchée par la malédiction collective »(Paré 1992 : 130), n'est- 
«Jusqu'à quel point ces symptômes aulturels jouent-ils au niveau individuel? » il pas abusif, excessif et même insoutenable de considérer «la folie furieuse et démente » rencontrée chez certains de leurs personnages comme représentative d'une conscience collective et surtout individuelle généralisée Franco- 0 ntarienne? Pourtant, ces mises en scènes rejoignent, il faut le dire, la terminologie tout aussi évocatrice de démence, du sociologue R oger Bernard qui parle de culture éclatée, d'embrouillement de frontières linguistiques et culturelles, de fragmentation irréversible, de désagrégation des sentiments d'appartenance, de métissage identitaire, de périphérisation de la francité, de dépossession culturelle et de situations d'impasse, d'obnubilation par le bilinguisme et le biculturalisme (1990; 1991a; 1991b; 1993; 1994; 1998). Et selon lui toujours, "nous sommes continuellement confrontés à cette dure réalité des « étrangers à nous-mêmes", conclue-t-il à la fin de son livre, L e C anada français: entre mythe et utopie (1998 : 213).

Jusqu'à quel point cessymptômesculturelsjouent-ils au niveau individuel? Loin de nous l'idée de se laisser emporter à poser un diagnostic médical et psychiatrique sur toute une population qui serait faite de générali sationsfaciles à partir d'un résultat d'enquête, de métaphores littéraires et d'une analyse sociologique particulièrement percutante. $\mathrm{N}$ ous avonsjugé bon de questionner desfrancophonesdeToronto aux prisesavec destroublesmentaux, pour connaitre leur vision des choses et explorer surtout les différentes trajectoires suivies en ce qui a trait à leur besoin ou non de services en français et aux barrières rencontrées pour I'obtention de ces services. II est temps de les écouter.

\section{Vingt-deux francophones nous parlent : qui sont-ils?}

À la demande du Conseil régional de santé, plusieurs agences, institutions et intervenants offrant des services de santé mentale à Toronto ont contacté des clients francophones avec qui ils avaient eu des contacts et les ont invités à participer à notre étude.V ingtdeux d'entre eux ont accepté d'être interviewés ${ }^{15}$. II y avait deux 
fois plus de femmes que d'hommes. Leur âge se répartissait entre cinq ans et 94 ans. Les trois enfants étaient représentés par leurs parents. II n'y avait pas d'adolescents ${ }^{16}$ et les adultes étaient âgés, en général, entre 35 et 64 ansalorsque six avaient au-dessus de 65 ans. Q uatorze sont $C$ anadiens Français: quatre sont originaires de I'O ntario, onze du Q uébec, un du N ouveau-B runswick. Lesautres, touscitoyens $C$ anadiens, viennent de laFrance, du Liban, de l'Égypte, du $\mathrm{M}$ aroc et d'H aïti. Ils étaient ici, en général depuis plus de dix ans, bon nombre ayant passé presque toute leur vie ici.

Au niveau de la langue comprise et parlée, vingt et un de nos répondants considèrent que leur français est excellent ${ }^{17}$. H uit considèrent que leur anglaisest excellent, et quatorze le considèrent comme étant soit pauvre (9) ou assez bien (5). Douze parlent uniquement ou principalement le français au cours de leurs activités quotidiennes. Cing parlent uniquement ou principalement anglais. D eux parlent les deux langues de façon égale et deux parlent le français, l'anglais et une troisième langue.

Clairement, il ne s'agit pas de présenter cette population comme représentative des divers segments constitutifs de la francophonie torontoise. II s'agit plutôt de communiquer à travers cestémoignages individuels révélateurs de besoins et d'expériences qui parfois se distinguent et bien souvent ont beaucoup en commun les uns avec les autres. $C$ 'est une population qui veut et doit absolument être entendue.

\section{De quels problèmes de santé mentale s'agit-il?}

Avant de mettre l'accent sur le rapport que nos répondants entretiennent avec la langue utilisée en thérapie, il importe de comprendre la gravité de leur «problème de santé mentale ». II importe de les connaître au moins un peu, malgré l'anonymat, pour bien les entendre.A ucun, il faut bien le dire, n'a directement associé son état de santé à sa condition de minoritaire, mais celleci, comme nousle verrons, est omniprésente dansleur conception 
« O n parle de schiz ophrénie, de dépression, d'angoisses, d'anxiété et de phobies, d'ennui, de solitude, de stress, de harcèlement, de drames familiaux et conjugaux, d'embolie, de séropositivité et de Sida, de paranoïa, de symptômes maniacodépressifs, d'instabilité émotionnelle et de tentatives de suidide. » de soi et revient inéluctablement au niveau du récit des difficultés rencontrées en thérapie. Les termes utilisés pour qualifier leur malaise entremêlent causes apparentes et effets, le physiologique, le relationnel, le situationnel et l'intensément personnel. «M oi, c'est la tête, la mémoire, le découragement ». O n parle de schizophrénie, de dépression, d'angoisses, d'anxiété et de phobies, d'ennui, de solitude, de stress, de harcèlement, de drames familiaux et conjugaux, d'embolie, de séropositivité et de Sida, de paranoïa, de symptômes maniaco-dépressifs, d'instabilité émotionnelle et de tentatives de suicide. "Q uelle vie! Ç a vaut rien, j'suis pas capable... J'ai prisun couteau etj'me suis coupé le cou...j'essayais de le rentrer mais pas assez fort et puis c'était noir et j'me sentais serré, fini, plus capable de vivre », nous confie ce vieillard arrivé en 0 ntario dans les années 50 . Et puis, «quand on se sent isolé, on oublie qui on est », dit une Q uébécoise dans la cinquantaine qui vit en 0 ntario depuisl'adolescence. $0 \mathrm{n}$ y retrouve toute une gamme de tragédies personnelles et humaines. «J'ai plus les symptômes majeurs, d'entendre des voix, paranoïa, d'me voir à la télévision... M ais j'ai encore l'angoisse intérieure », révèle cette quadragénaire en 0 ntario depuis plus de vingt ans... ou encore, «M on psychiatre pensait que c'était parce quej'étais homosexuel, mais c'est parce que mon amant venait de me quitter ", déclare cet homme dans la trentaine, ici depuis une dizaine d'années. Parfois, on se veut plus discrète sur la nature de ce qui nous a traumatisée: "J'ai été élevée dans des circonstances émotionnellement et psychologiquement très dures ». Parfois, on évoque le déclenchement inattendu, foudroyant: "J'étais une enfant sage. À l'adolescence, tout a éclaté... ». Pour les enfants, on attribue les problèmes à «l'hyperactivité » ou à «l'épilepsie ».

Puis, il y a les drames familiaux («Tout a commencé quand mon fils ... ») ou les déchirures («A près la mort de mon enfant, j'ai fait une très grosse dépression, je ne pouvais plus bouger ou rien faire ») et les déceptions profondes face à sa vie et son travail ( $C$ C 'était la frustration de vivre au niveau où je vis et de travailler dans des domaines qui ne sont pas nécessairement stimulants intellectuellement alors que je connais mes capacités qui sont très grandes »). 
Enfin, tous peuvent dire quand les problèmes ont débuté, soit récemment (dans 13 cas, il y a moins de cing ans) ou il y a fort longtemps (pour trois, il y a plus de 16 ans), dans leur région natale (9) ou àToronto (13).

\section{Langue parlée et langue préférée en thérapie}

«... et puis les services en français, ils devraient avoir des gens qui parlent français au moins à chaque endroit plutôt que d'avoir un endroit préas où on doit aller pour des francophones, »
Q uinze de nos répondants préfèrent ou préféreraient nettement recevoir des services de santé en français; cinq autres disent qu'ils n'ont pas de préférence, une personne est incertaine et une n'en veut pas. Pourtant seulement six personnes sur vingt- deux parlent français avec leur intervenant principal, au moment de l'enquête. Ce n'est donc pas uniquement parce qu'on n'en veut pas que I'on n'a pas de services en français! Comme nous le verrons cidessous, certains ne le demandent pas, d'autres se battent pour en avoir et ne rencontrent que refus, $d$ 'autrescherchent en vain. $R$ ares sont ceux et celles à qui I'on en a offerts spontanément. Seules trois personnes se rappellent que quelqu'un ait priscette initiative: une à l'urgence, une par une infirmière et un par un médecin de famille francophone qui voulait la référer à un psychiatre. U ne d'entre elles, par contre, se dit offusquée qu'on lui ait offert des services en français, car elle y voyait une façon de la contrôler, de se débarrasser d'elle et de limiter ses choix. Bref, elle y voit une marginalisation, exclusion, «ghettoïsation »et n'aime pasça : «J'me sens pas chez moi en me faisant dire ça! Si j'suis là, c'est parce que je veux l'être et puis les services en français, ils devraient avoir des gens qui parlent français au moins à chaque endroit plutôt que d'avoir un endroit précisoù on doit aller pour desfrancophones».

Les raisons pour lesquelles on ne demande pas de services en français varient énormément. $0 \mathrm{n}$ n'en veut tout simplement pas: "quand on vient rester dans un paysanglophone, on doit s'intégrer. $C^{\prime}$ 'est pas à eux de s'intégrer. C'est à nous de s'intégrer [... ] 0 n vient ici pour s'intégrer $» .0 \mathrm{u}$ encore, on n'a pas de nette préférence, donc, on ne voit pas pourquoi demander: "M oi, l'anglais pis le français, c'est pareil pour moi, y a pas de différence... 
M on anglais est $100 \% » .0 \mathrm{u}$ encore, lorsqu'on vit en 0 ntario depuisl'âge de 13 ans, le français, pour ses soins en santé mentale, «n'est pas vraiment nécessaire [... ] A près cinquante ans de vie bilingue, je ne pense pas qu'il y ait des choses que je préférerais exprimer dans une langue ou une autre. Les deux langues sont là pour que j'exprime ce que j'ai à exprimer ». Partie du Q uébec pour l'O ntario, il y a déjà plus de 75 ans, une répondante par contre, aurait aimé avoir des intervenants francophones pour l'aider avec sa schizophrénie, mais «c'était déjà trop demander », dit-elle. Elle n'aurait pas osé :

D e toute façon [... , j' étais tellement heureuse d' avoir un peu d'aide. J'me suis dit, il faut bien être conne: j'arrive quelque part, ils m'accueillent sur une autre planète, est-ce qu'il faut que je demande en plus des soins particuliers? Bien, déjà que c'était beau, j' ai pas pensé que je recevais pas assez. Puiş, je m'suis mise à apprendre l'anglais un peu mieux. [... ] M aintenant, c'est tellement naturel de penser en anglais que j'y pense plus

De plus, maintenant, il faudrait se réadapter, et cela serait trop difficile, alors elle ne demande toujours pas: «Y a tout un vocabulaire de santé mentale que j'ai appris en anglais, puis maintenant, le dire en français, j'aurais des problèmes. Faudrait que j'regarde dans un dictionnaire ».

« L'embarras, la gêne, la confusion, le dépayse ment lorsqu' on se trouve à I'hôpital jouent aussi un rôle important. »
L'embarras, la gêne, la confusion, le dépaysement lorsqu'on se trouve à l'hôpital jouent aussi un rôle important. U n homme de plus de 70 ans nous dit: "J'ai pas demandé de services en français parce que ça m'embarrassait d'être là [... ] J'ai pas demandé parce que je savais pas où j'étais rendu ». Puis, cela aurait demandé trop d'énergie, énergie qu'en ce moment, après sa tentative de suicide, il n'avait pas: " J'étais faible un peu, non, j'ai pas demandé de services en français [...] On ne sait pas ce qu'on fait, on est découragé, ça fait comme si on a les mains molles [... ] $0 \mathrm{n}$ n'a pas de force. Puis d'la tête, on en a pas, on est perdus [... ] ». M algré son anglais vacillant, son «jargon», comme il dit, on ne lui a pas offert d'interprète. 
«II y a également... les règles de la politesse, bien endoctrinées surtout celles qui gèrent le comportement du minoritaire face au majoritaire, du vainar face au vainqueur. »
«... d'autres ont

l'impression de se battre perpétuellement et ce, sans grand succès, »
Il y a également, et nous en connaissons tous le pouvoir, les règles de la politesse, bien endoctrinées, surtout celles qui gèrent le comportement du minoritaire face au majoritaire, du vaincu face au vainqueur. U ne Q uébécoise dans la quarantaine, en 0 ntario depuis cinq ans et demi, rencontre finalement en thérapie de groupe une autre cliente francophone qui, pourtant, refuse de lui parler en français, «par respect pour les autres ». « Pourtant, ditelle, on ne se crie pas à toute tête en français, on se parle! ».

Pour d'autres, enfin, ce n'est pas qu'on ne demande pas, c'est qu'on ne demande plus. O n capitule, saisis d'un sentiment de frustration :

$0 \mathrm{n}$ a beaucoup de mal, à tous les niveaux, du gouvernement, à tous les niveaux, de se servir du français. $0 \mathrm{n}$ se moque de nous, on nous prend pour des idiots $E$ t surtout, jamais dans un hôpital, on n'a jamais été accueillis en français, alors, on ne demande même plus car on nous regarde comme des animaux étranges venus d'ailleurs C 'est sûr qu' on en souffre

Alors que certains ne demandent pas, ou ne demandent plus, d'autres ont l'impression de se battre perpétuellement et ce, sans grand succès. Q uébécois demeurant à Toronto depuis une douzaine d'années, il est âgé dans la trentaine, est séropositif et souffre, dit-il, de «dementia ». II a beaucoup de difficultés à vivre àToronto. "C'est un combat constant [... ]», ceci le rend encore plus malade.

À vivre id à Toronto et à me battre tous les jours pour avoir des services dans ma langue me rend dépressif. Ç a sert à rien pour moi de voir un psychiatre en anglais parce qu'il y a des termes que je ne comprends rien, puis j'peux pas m'exprimer. C e qui vient chercher en dedans de moi, au plus profond, c'est ma langue maternelle [... ] Je cherche quelqu' un, un psychiatre francophone avec qui je peux me vider le cour, mais je ne trouve pas 
Partout, il ne rencontre que résistances et refus:

O n m'a partout répondu:You speak English quite good [... ]. Ben, je n'en ai eu auaun service, pas du tout de services en français, j'ai eu aucun service. $0 \mathrm{n}$ $m$ ' offre toujours des services d' anglophones, je ne peux pas m'exprimer assez bien [... ] A lors je refuse d'y aller [... ] Ç a devient pour moi un cauchemar! [... ] Ç a me tue de pas avoir les services en français dont j'ai besoin.

II s'est adressé à l'Église pour de l'aide. M ême là, dans sa propre communauté, il n'a pasété bien reçu : «Y 'aune église francophone dans le quartier. J'ai demandé de l'aide à cette église là. $\mathrm{O} n$ ne voulait pas m'aider. On m'a dit : on ne vous voit pas souvent à l'église, on ne vous connaît pas, vous pourriez être un voleur, on ne peut pasvousaider ». Défait, il a trouvé sa solution : «Je retourne au Q uébec pour avoir des services garantis en français ».

$Q$ uébécois d'origine et homosexuel, un autre répondant est en 0 ntario depuis huit ans. II fait une tentative de suicide après avoir vu deux psychiatres anglophones qui selon lui «étaient homophobes » et avaient des idées préconçues sur son mal. II précise que : «d'avoir pu m'exprimer en français aurait prévenu ma tentative de suicide ». II cherche toujours, sans trouver.

J'ai toujours des commentaires lorsque j'essaie de m'exprimer en français ou de recevoir des services en français. Ils me disent tu peux parler anglais un peu, t'es capable det' ex primer en anglais Tu dis : oui, j'suis capable de m'exprimer en anglais pour te dire qu'je suis malade, mais pas pour expliquer les problèmes exacts quej'ai. J'peux pas le faire en anglais parce que j'connais juste les termes en français

O n se rappellera ici d'un rapport sur la même problématique présenté par l'A ssociation canadienne pour la santé mentale en 1987 pour la région 0 ttawa- $C$ arleton et ayant comme titre percutant, Q uand je suis malade, je ne suis pas bilingue (1987). C'était vrai hier, c'est vrai aujourd'hui. 
«II arrive souvent que la demande de services en français soit comprise comme une exigence de nature strictement idéologique ou politique... »
Parfois l'attitude rencontrée est hostile: "Ils ne nous aiment pas beaucoup lesA nglais! », dit I'une. II arrive so uvent que la demande de servicesen français soit comprise comme une exigence de nature strictement idéologique ou politique : «On m'identifie très vite au Q uébec. Certaines personnes ont des préjugés au sujet des Q uébécois! ». Le risque est toujours là et lorsqu'on est vulnérable, on ne peut pas se payer d'attiser la rancœur, la xénophobie.

$M$ ais les barrières ne se limitent pas à cette attitude réfractaire, si souvent rencontrée. Parfois, on cherche, on trouve, mais «ces services sont trop loin "; "c'est pas à notre portée », nous dit cette femme seule; "c'était le soir et je ne voulais pas faire le trajet par métro; je conduis pas beaucoup » nous explique une autre ou encore, ce n'est pas ce dont on a besoin : "on a pas assez de portes de sorties pour aller chercher du secours; à M ontréal, j'avais toutes les sorties. $Y$ 'a pas le service dont on a besoin dans les écoles françaises", nous dit un père émigré d'outre-mer il y a quelques années et dont la petite fille d'âge scolaire doit demeurer à la maison et apprendre l'anglais deux heures par jour, aux frais de la commission scolaire anglophone du secteur afin qu'elle puisse intégrer leurs classes spéciales. "J'aime autant oublier le système français», conclue-t-il.

Finalement, on ne sait pas où chercher, où trouver : "J'aurais aimé un médecin francophone, mais où le trouver? » est une question entendue maintesfoislors de nos entrevues. Le sentiment "de ne pas avoir les outils nécessaires», «de frustration », «d'impuissance », est intolérable.

\section{L'expérience des services en anglais lorsqu'on n'a pas le choix : «torture, cauchemar..."}

L'absence de service en français s'avère être une véritable tragédie pour bons nombres de nos répondants. Leurs témoignages sont fort révélateurs de situations intenables et souvent extrêmement dangereuses D eux nousont dit : «Je suisune bombeà retardement ». 
N ous les avons tous laissés parler, s'ouvrir le cœur, en français : «0 n m'a traitée plus différemment que j'étais différente ». Parfois I'intervenant fait un effort, mais pour un temps seulement:

Q uand il y avait des situations angoissantes quand je cherchais mes mots, il essayait de me corriger, de m' aider [... ] J'aurais aimé une personne avec qui je pouvais parler. M aintenant, il ne fait pas de psychothérapie, il prescrit des médicaments $0 \mathrm{n}$ ne parle pas beaucoup. C 'est surtout pour me prescrire des médicaments [... ] $M$ on mari me reproche d' avoir une vraie pharmadie dans ma chambre [... ] ]'ai dû aller récemment dans un foyer de désintoxication afin de prendre moins demédicaments

Et puis, pour certains, mieux vaut sortir de là :

Je vous le jure, madame, quand j'étais malade, j'ai passé proche de mourir. Je faisais une maladie qu' on appelle dementia et $c^{\prime}$ est extrêmement difficile de demander à quelqu' un qui parle une autre langue de parler une autre langue. [... Pendant deux semaines, les médecins me disaient quelque chose, ça me rentrait pas du tout dans la tête et j' ai rien compris de ce qu'ils disaient [... ] J'ai perdu du poids parce que personne ne me comprenait quand je disais ce que je voulais manger pour vivre. J'ai dit au lieu de rester à l'hôpital pour mourir, je vais à la maison pour vivre Je serais resté à l'hôpital, je serais mort.

Le stress en rend un autre encore plus malade :

J'peux aller magasiner en anglais, mais pas parler de mes émotions [... ] Je peux y arriver avec mon gynécologue, mais lorsqu'il s' agit d'émotions et de santé mentale, cela m'est impossible, c'est un stress épouvantable de devoir le faire en anglais. Je l' ai vécu et ça me rendait malade, encore plus malade.

Au moins, l'anglais est une langue humaine, cela réconforte :

C 'était en 72 et c'était en anglais C a m'a étonnée, j'me suis dit, tiens, un semblant d'humain. J'avais 
pris des cours d' anglais à l'école et je savais que c' était une langue humaine, mais j'la comprenais pas $M$ ais tout d' même, j'savais qu'il y avait un peu de familiarité là-dedans, c'était d'la même espèce. À part ça, tout était étrange [... ] ]'savais que c' était une autre planète.

Et puis, certaines personnes sont oubliées et s'enfouissent dansles rues. Au poste de police qu'elle avait appelé pour de l'aide, en arrivant sur le pouce avec son enfant àToronto, une jeune mère réussit à communiquer avec «le langage des signes appris au Q uébec! ». Le jour même, une fois son enfant en sécurité avec l'Aide à l'enfance, elle tente de se jeter en bas d'un pont.

À l'hôpital, tout s'est passé en anglais J'en ai demandé (des services en français) mais ils ont dit qu'il n'y en avait pas. $0 \mathrm{n}$ m'a laissée pour acquis là. $0 \mathrm{n}$ m'a foutu dans un lit, je faisais partie des meubles $0 \mathrm{n}$ pouvait pas communiquer avec moi. $0 \mathrm{n}$ m'a pas offert d'interprète

D eux semaines plus tard, elle sort de l'hôpital pour prendre un peu d'air frais et se perd dansToronto. «Je n'ai pas pu retrouver I'hôpital ». Enceinte de son deuxième enfant, elle passe les prochains six mois de sa grossesse dans les rues, sans abri. « $C$ 'est frette Toronto, I'hiver! ». Au moment d'accoucher, elle appelle l'urgence...

Certains sont plus chanceux, dirait-on. Ils ont un conjoint, une mère qui peut les aider à traduire ou agir comme interprète, situation loin d'être désirable ou confortable.

(L e psychiatre) était un homme très compréhensif, très doux, très calme [... ] M on gros stress, c'était qu'avant de partir, j'demandais à mon mari qui, lui, est parfaitement bilingue, comment tu dis ça en anglais $P$ uis là, je préparais mes entrevues avec le psychiatre d' avance avec mon mari en demandant les termes en anglais A lors, cétait une torture A lors, j' pouvais vraiment pas guérir parce que chaque fois que j'voyais le psychiatre, même s'il était extraordinaire, le fait pour moi, surtout malade comme je l'étais, d'avoir à dire mes problèmes 
émotifs en anglais, c'était une torture. ( $M$ a fille) fait ce qu'elle peut. Q uand la thérapeute lui parle, elle va répondreen foncion decequ' ellea compris et très souvent, elleva venir mevoir et medemander : maman, comment dit-on telle chose en anglais A lors, je traduis

« L a thérapie de groupe, en anglais, $s^{\prime}$ avère souvent un véritable supplice... »
La thérapie de groupe, en anglais, s'avère souvent un véritable supplice, comme en témoigne une Acadienne àToronto depuis 20 ans :

L a thérapie de groupe, en anglais, ça me rendait malade Ç a m'aidait pas dans l'état où j'étais [... ] J'me culpabilisais tellement, j' me trouvais tellement stupide, de pas parler anglais [... ] Ç a me bloquait, me stressait. J'avais mal au ventre avant d'aller, ça me rendait encore plus malade. Je voyais tout ce monde qui parlait en anglais. J'les enviais, alors moi j' me démolissais, j'suis donc stupide, j'arrive même pas à parler couramment anglais comme eux. Ç a m'emmerdait énormément.

Et elle ajoute :

[... ] j'les vois pleurer, bon, j'comprends qu'ils sont [... ] upset [... ] parce que ça pleure. $M$ ais œ qu'ils vivent à l'intérieur, j'le ressens mais j'comprends pas le pourquoi [... ] F ait qu'à kek part, j'me sens perdue J'suis dans un groupe où je vois plein d'émotion, puis j'arrive pas à comprendre le pourquoi [... ] P uis, quand c'est mon tour à parler, j'leur en veux pas parce que j'ai appris à rire de mes erreurs que j'fais en anglais, sauf que c'est frustrant dans un autre sens parce que j'essaie de dire kek chose, pisj' cherche une compassion kek part et tout cej'ai comme feedback, c' est des pouffées de rires

$M$ ais, nous dit une autre répondante, qui parle souvent tout haut tout seul en public, il y a tout de même un avantage à ne pas être compris:

J'ai arrêté d'me retenir. À quoi ça sert. J'ai quelque chose à me dire, j' me le dis. Puiş j'va te dire, souvent, ça 
sort en français A lors, c'est pas embarrassant, la plupart comprennent pas [... ] Puis, quand je veux un siège dans le métro, jememets à parler ainsi en français et j' ai trois sièges à moi toute seul: mais essayer d'avoir l'air folle, tu peux avoir l'air fou. Faut l'faire avec dasse!

\section{L'expérience des services en français : succès parfois bien mitigé!}

«P ersonne ne prend les services en français pour acquis simplement parce que la L oi 8 leur en donne le droit. »
Certains trouvent finalement un psychiatre francophone par l'entremise de l'annuaire francophone, une infirmière qui parle français dans un hôpital et qui agit comme guide, le Centre médico-social de Toronto, SO S femmes, le Centre H éritage, le Centre des Pionniers, et même le $C$ entre francophone qui offre de bons conseils. Ils trouvent parfois en utilisant des moyens tout à fait inusités. U n de nos répondants, entre autres, s'est adressé au B loc Q uébécois pour leur demander de lui trouver un psychiatre francophone àToronto, ce qu'ils ont fait. N os répondantsattribuent généralement cette « découverte » à la «chance », et, de fait, ils se considèrent nettement «chanceux $»$. Personne ne prend les services en français pour acquis simplement parce que la $L$ oi 8 leur en donne le droit.

U ne F rançaise, merd mon D ieu! J'avais un médeain extraordinaire, puis elle était canadienne française, à part ça. J'ai été chanceuse. (J'avais été) barrouettée de gauche à droite mais enfin, on (S.0.S. femme) $\mathrm{m}$ 'a trouvé ce médean-là. $C$ 'est une femme extraordinaire [... ] S.O.S. femme, une chance que j'les ai eus, je serais bien folle aujourd'hui.

Avoir finalement trouvé le nom d'un psychiatre francophone dans I'annuai re francophone deToronto et qu'il ait accepté de la recevoir sans référence de la part d'un généraliste, pour une autre, «c'est le plus beau cadeau de la terre [... ] Alors pour moi, c'était plus qu'un million! Sa réponse était oui! Parce que je sentais que j'étais 
«E t puis le français parlé par les interve nants... laisse parfois à désirer. ... « Je sais qu'ils $s^{\prime}$ essaient beaucoup $[\ldots]$ ça doit être diffiale pour eux mais, je sais pas pourquoi, c'est plus fadile pour moi de parler anglais que pour eux de parler français ». » en train de me noyer. Puis quand j'ai vu ce psychiatriste (sic) là, ça été le bois d'épave auquel je me suis accrochée pour pas me noyer $»$.

Pourtant, l'expérience n'est pas toujours satisfaisante. 0 n est parfois profondément déçus. «J'ai pas été satisfaite de l'effort de l'école (francophone, en psychopédagogie), c'était pas assez complet ». Parfois on se rend compte que le service pourtant francophone n'est pas adapté à ses besoins: " C'est des fausses promesses! [... ] J'peux pas parler, elles sont toutes sourdes! Sont toutes à moitié mortes sur une chaise roulante [... ] C 'est platte! Je fais rien, absolument rien ici ».

Et puis le français parlé par les intervenants dans certaines institutions, même accréditées, laisse parfois à désirer. Alors, on fait la seule chose polie et possible:. " Je sais qu'ils s'essaient beaucoup [... ] ça doit être difficile pour eux mais, je sais pas pourquoi, c'est plus facile pour moi de parler anglais que pour eux de parler français». Et ajoute-t-elle à propos d'une institution où on offre desservicesen français, «A u moinsici si je disun mot en françaiscar je le trouve pas en anglais, ils vont le comprendre! ». L'expérience est assez courante, on s'y reconnaît facilement : «J'ai finalement trouvé un intervenant qui parle français, mais quand j'ai réalisé que son français était pire que mon anglais, j'ai décidé de parler anglais, pour lui aider ».

Enfin, rien n'est garanti à vie. Après avoir passé des mois, enceinte, dansles rues deToronto, cette jeune mère de deux enfants maintenant, avait trouvé. Et pourtant:

ma plus grosse crainte est dans le temps des fêtes, quand il y a eu des coupures dans les services sociaux, ça a été de perdre ma thérapiste. Ç a a eu un gros impact sur moi, sur ma qualité de vie avec mes enfants. $M$ on insécurité s' est manifestée par de l' impatience, manque de sommeil, nervosité [... ] des crises de larme. Pour I'instant, jeneme verrais pas vivre un avenir rapproché, sans thérapiste. Je dégénérerais rapidement.

Spécifions ici que les personnes interviewées avaient toutes fait appel à des services publics de santé mentale, c'est ainsi qu'on a 
pu les repérer. Pourtant, si on se fiait uniquement aux données du Supplément de Santé $M$ entale de l'enquête sur la Santé de l'O ntario examinées en première partie de cet article, elles auraient donc toutes trouvé une réponse à leurs besoins de services. N ous sommes bien loin de la réalité!

\section{Et la famille, le réseau, la communauté, l'aide naturelle?}

«P resque personne

n'avait, dans la communauté, quelqu'un avec qui «se vider le cour » comme ils |'auraient tant désiré, »
D ans presque tous les cas, les adultes étaient isolés, seuls dans la vie. "J'ai perdu ma dignité, mon emploi, mes amis», nous dit l'un. «T'es vu comme un crack pot, une personne qui abuse des drogues [... ] U ne personne qu'il faut se tenir de loin », nous dit un autre. «Q uand j'avais besoin de parler avec quelqu'un, on me disait qu'on avait pas le temps, alors je me disais que ce ne sont pas des amis", renchérit-il. Presque personne n'avait, dans la communauté, quelqu'un avec qui «se vider le cœur » comme ils l'auraient tant désiré. Ils ne savaient pas trop de quoi on parlait lorsqu'on mentionnait leur "communauté culturelle». "Je ne fais partie de rien » est la réponse générale. «Elle est où, cette communauté francophone? », nousont demandé plus d'uns, « tout est éparpillée! » ont déclaré les autres. Côté religion, ils disent souvent avoir une certaine foi, mais font rarement partie d'une Église. Bien que certains disent avoir confiance, si nécessaire de trouver là du support, le seul qui nous a dit l'avoir fait, est retourné bredouille. U n autre énonce catégoriquement «L'Église, je ne voudrais pas du tout qu'ils sachent que j'ai des problèmes de santé mentale! ».

Souvent leurs enfants, leurs aidants naturels, ne les comprenaient pas. «D onnez, donnez [... ], mais ne recevez rie », dit une mère qui se sentait abandonnée. «M a famille? », dit une autre, « $\mathrm{N}$ on! c'est pas du méchant monde. Ils ont leur petite vie et ne veulent pas être dérangés avec des gros problèmes comme moi ». 


\section{Ce que vous aimeriez plus que tout au monde?}

«M ais surtout, on voudrait tellement une personne avec qui parler vraiment, «sur qui compter, se défouler » dans sa langue »
Q uand on leur demande quels services ils aimeraient plus que tout au monde, pris par surprise, nos répondants expriment d'abord des désirs bien modestes et presque instinctivement reliés à une meilleure qualité de vie: un bain plus que deux fois par semaine, de la meilleure nourriture et la messe en français tous les jours si on réside en institution, de l'aide pour lire (" pour être plus indépendant, car les yeux déclinent $»)$, des services accessibles pour la chaise roulante et quelque chose à faire de ses journées! U ne autre répond spontanément: «U n million! ». Puis, on y réfléchit, et chacun y ajoutant du sien, une longue liste de désirs bientôt apparaît; on mentionne le psychiatre, le psychologue, le travailleur social, le psychopédagogue pour ses enfants, l'infirmière visiteuse et le pharmacien qui «peut aussi sauver des vies » ou encore, pour plus d'accessibilité, une ligne téléphonique d'information pharmaceutique. On mentionne surtout le groupe thérapeutique ou groupe de soutien, pour contrer la solitude et partager. «C e serait merveilleux », tout en français et accessible. $0 \mathrm{n}$ parle de programmes de réentraînement au travail, de programmes de jour et de services de répit pour les parents, de services d'urgence. C ertains voudraient de l'ai de pour faire le magasinage et même l'ouvrage de maison lorsqu'on ne s'en sent pas capable. On souhaite des intervenants qui comprennent la problématique homosexuelle, multiculturelle, immigrante et réfugiée. $U$ ne répondante nous raconte qu'elle fait des hallucinations ancrées dans sa culture d'origine que le psychiatre ne connaît pas. 0 n suggère la création d'un bottin ou d'un annuaire francophone de tous les services dont une personne dans leur situation aurait besoin «comme ça, on saurait où chercher »; de même qu'un système de transport qui pourrait les mener là où sont les services, étant donné les longues distances à parcourir.

$M$ ais surtout, on voudrait tellement une personne avec qui parler vraiment, « sur qui compter, se défouler » dans sa langue. 
«U ne personne, comme vous», dit-on, «en qui on a confiance, avec qui on peut s'exprimer ». Puis, avec un brin d'espoir: «s vous revenez, je parlerai encore! ».

\section{Conclusion et réflexions}

«E ntre droits acquis et services regus, cependant, doit s'insérer tout un travail de planification et de réalisation tant de la part des instances gouvernementales, institutionnelles et communautaires que des intervenants et même des réaipiendaires »

« Les défis sont énormes tant au sein de la communauté qu'au niveau du système... »
Depuis 1986 , suite à la L oi 8 sur les Services en français de I'O ntario, les francophones souffrant de problèmes de santé mentale dans toutes les régions désignées ont le droit de recevoir les services dont ilsont besoin en français. Entre droits acquis et services reçus, cependant, doit s'insérer tout un travail de planification et de réalisation tant de la part des instances gouvernementales, institutionnelles et communautaires que des intervenants et même des récipiendai res. Le but de cet article était de présenter un portrait, tant épidémiologique que profondément humain de ces francophonesqui, àToronto, rencontrent toujours de solides barrières à l'obtention de services, doivent encore « se battre », «chercher sans être certains de trouver » et compter sur « la chance » pour avoir réponse à leurs besoins en français. II est clair que les francophones aux prises avec de tels problèmes ne sont «pas encore servis adéquatement » comme le dit si bien le document de la réforme initiée en 1993. Loin de là! Les défis sont énormestant au sein de la communauté qu'au niveau du système : fragmentation géographique et vaste diversification ethnoculturelle de la communauté, dispersion et inaccessibilité des services sur un territoire étendu, gamme extrêmement limitée de services, manque de personnel francophone qualifié, de facteurs incitatifs pour en attirer, en former, budget non protégé, coupures gouvernementales qui sabrent au cœur même des services, absence de communication, de leadership et de partenariats vivifiants entre services et agences désignées, promesses non tenues, manque d'intérêt de la part des intervenants, attitudes préjudiciables et parfois hostile envers les francophones, manque d'information tant chez les intervenants anglophones que francophones à savoir où trouver... À l'aube de I'an 2000, l'heure est à la réforme et les francophones devront 
compter sur une volonté politique convaincue, courageuse, déterminée et omniprésente de se conformer aux exigences de la L oi 8. C'est une arme pourtant bien faible et vulnérable lorsque viennent s'interposer, surtout àToronto, le jeux puissant desnombres et les discours sur le partage équitable des ressources entre groupes ethnolinguistiques. C'est là, le plus grand défis, car les besoins sont énormes de toutes parts, les mémoires historiques courtes et la compétition féroce. Ainsi, il n'en tient qu'à une phrase, la seule sur les services francophones glissée au terme d'un paragraphe sur la démographie de Toronto, dans un document de discussion du C onseil R égional daté du moisd'août 1998 et intitulé Implementing the M ental $H$ ealth System D esign :

The top five « home language » spoken in Toronto are E nglish, $\mathrm{C}$ hinese, Italian, Portugese and Spanish. In addition, $25 \%$ of the residents reported a « home language » other than E nglish or F rench, the two official languages It is well recognized that culture plays a significant role in the way mental health problems are perceived and handled.T he aty ofToronto is designated to provide services to the $F$ rancophone community under the $F$ rench $L$ anguage Services A ct (1998: 2).

$M$ alheureusement, le bassin de population francophone est minime en comparaison. Un document subséquent dont le but est de décrire la stratégie de partenariat entre le Conseil régional et le $M$ inistère en vue de la mise en application du système de santé mentale de Toronto se fait plus discret, silencieux même, sur la $L$ oi 8 , mais communique fort bien la nature de l'obligation, de la difficulté et de l'embarras.

$\mathrm{H}$ andling the implementation related activities in the transition phase will be partiaularly difficult since the system manager must be seen to be fair and impartial, while at the same time adhering to all the polides to which the system players are subject (e.g. the policy and principles outlined in Putting the People F irst) (s.d.: 19)

Voilà donc où nous en sommes... à l'aube de l'an 2000. 


\section{Bibliographie}

ASSO CIATION CANADIEN NE-FRANÇAISE DE L'ONTARIO (ACFO) (1989). Les F rancophones tels qu'ils sont. $R$ egard sur l'O ntario français à l'aube de la $L$ oi de 1986 sur les services en français O ttawa,ACFO.

ASSO CIATION CANADIEN NE POUR LA SANTÉ MENTALE (ACSM) (I987). When I'm Sick, I' $m$ N ot Bilingual: Position Paper on the Planning of $F$ rench $L$ anguage $M$ ental Ù health Services for $A$ dults $B$ etween the $A$ ges of 16 to 65 , Section $O$ ttawa- $C$ arleton ( $N$ ovembre).

AMERICAN PSYCHIATRIC ASSO CIATION (I987). D iagnostic and statistical manual of mental disorders (T hird edition - revised. D SM - III-R ),Washington, D.C,A merican Psychiatric A ssociation.

BAVIN GTO N J. ET A. M AJID (I986). «Psychiatric Servicesfor Ethnic M inority Groups», in John L.C ox, Transcultural Psychiatry, G reat B ritain,Biddles Ltd, Guildford and K ing's Lynn

BEISER, M . (1988). R eview of the Literature on M igrant M ental H ealth, ( C anadian Task Force on M ental H ealth Issues Affecting Immigrants and R efugees, ) C anada, M inistry of Supply and Services $C$ anada

BER N A R D, R . (I990). L e dédin d'une alture $R$ echeche, analyse et bibliographie sur la $F$ rancophonie hors $Q$ uébec, 1980-1989, 0 ttawa, Fédération des jeunes $C$ anadiens français (FJCF).

BER N AR D, R . (1991a). U n avenir incertain. C omportements linguistiques et conscience aulturelle des jeunes $C$ anadiens français, $O$ ttawa, Fédération des jeunes français.

BER N AR D, R (1991b). Le travail et I'espoir: migrations, développement économique et mobilité sociale $Q$ uébed 0 ntario, 1900-1985, H earst, Le N ordir.

BER N AR D, R . (1993). «La Culture éclatée », L iaison, no. septembre.

BER N AR D, R . (1994). L a question identitaire au C anada francophone. R édts, parcours, enjeux, hors-lieux (Jocelyn Létourneau, dir; R oger Bernhard, col.), Q uébec, PU L.

BER N AR D, R . (I998). L e C anada F rançais: entre mythe et utopie, Hearst, Le N ordir.

BLAN D et OR N dans Beiser , M ., (I981). R eview of the L iterature on M igrant M ental H ealth, C anada, $M$ inistry of Supply and Services

BO U D R EAU, F. (1996). T he N eeds of F rancophones with Severe M ental H ealth Problems in M etropolitan Toronto: " I $O$ nly $H$ ave theW ords in F rench ", a report of the French- Speaking $M$ ental $H$ ealth Q ualitative N eeds-B ased A ssessment Study Committee,Toronto, M etropolitan Toronto D istrict Health Council.

CAR R IÈR E, F. (I993). «La métamorphose de la communauté franco-ontarienne, 1960-1985 », dans Cornelius). Jaenen (s. la dir. de), L es F ranco- 0 ntariens, 0 ttawa, LesPresses de l'U niversité d'O ttawa, 305-341.

CONSEIL RÉGIONAL DE SANTÉ DETORONTO (S.D.) A Strategy for MTDHC/MOH to Implement the $M$ etroT oronto $M$ ental $H$ ealth System, A discussion Paper

CO N SEIL R ÉGIO N AL DE SAN TÉ DETO RO N TO (1998). I mplementing the M ental H ealth System D esign forToronto. A discussion Paper, August 1998.

DEM AN GEAT, M . (1989). «L'autre langue comme symptôme », P sychiatrie F rançaise, no mai, 73-75.

DESBIEN S, P. (1977). L es conséquences de la vie, Sudbury, Prise de Parole.

DESBIEN S, P. (1978). L'espace qui reste, Sudbury, Prise de Parole.

DESBIEN S, P. (1981). L'homme invisible/ T he Invisible M an, Sudbury, Prise de Parole et M oonbeam, Penumbra Press. 
H AVA SSY, B.E. et J.T.H O PK IN (1989). «Factors predicting utilization of acute psychiatric inpatient services by frequently hospitalized patients $», \mathrm{H}$ ospital and C ommunity P sychiatry, 40, 820-823.

LAN GELIER , R . (I982) «French C anadian Families» in M. M cGoldrick, J.K. Pearce, J. G iordano, E thnicity and Family T herapy, N ew York, The Guilford Press, 229-246.

LEO N G , F.T. (1986) «C ounseling and psychotherapy with A sian A mericans: review of the literature », Journal of C ounseling Psychology, 33, 196-206

LIN D SEY, K.P. and G. L. PAU L, (1989) «Involuntary Commitments to public mental institutions: issues involving the overrepresentation of Blacks and assessment of relevant functioning », Psychological Bulletin, 106, 171-183

M IN IST ÈR E DE LA SANTÉ (1993). Putting People First: T he R eform of M ental $H$ ealth Services in $O$ ntario/ M ettre la personne au premier plan: L a réforme des services de santé mentale en 0 ntario, Toronto, $Q$ ueens Printer for the Government of $O$ ntario

M IN IST ĖR E DE LA SANTÉ (1995). Implementation Planning G uidelines for M ental H ealth R eform, Toronto, Q ueen, Printer for the Government of O ntario.

M IN IST ÈRE DE LA SANTÉ (1995b) M ental H ealth Supplement: U ser's M anual, Volume 1: D ocumentation.

N EIG H BO R S, H .W. (1984) «Professional help use among B lack A mericans: implicationsfor unmet need », A merican Journal of $C$ ommunity P sychology, 12, 551- 566

PAR É, F. (1992). L es littératures de l'exiguïté, H earst, Le N ordir.

PAR É. F. (1994). T héories de la fragilité, Essai, H earst, le N ordir.

PASSAM AN ICK , B. (1963) «A survey of mental disease in an urban population :An approach to total prevalence by race », A merican Journal of Psychiatry, no 119, 2999-3054.

SH EFFLER , R .M .\& M ILLER ,A.B. (1989). «D emand analysis of mental health service use among ethnic subpopulations », Inquiry, 26, 202-215.

SN OW, C. (1987). «Le taux élevé d'internement dans le N ord du N ouveau-Brunswick », É galité, no 21, printemps, 81-87

SN OW DEN , L.R .\& CHEU N G, F.K (1990). «U se of inpatient mental health services by members of ehtnic minority groups $»$, A merican Psychologist, 45, 347-355.

ST-AM AN D, N . (1985). F olie et oppression, M oncton, Les É ditions d'A cadie L tée.

ST-AM AN D, N . (1992). «U ne A cadie troublée: santé mentale ou marginalisation des démunis? », N ouvelles Pratiques Sodales, vol. 5, no 1, printemps, 147-158.

ST-AM AN D \&, N .,V U O N G, D. (1994). «Q uand lalangue fait une différence. C e que des «bénéficiaires » pensent du système de santé mentale », Sociologie et Sociétés,Vol. X X VI, 1, 178-196.

WAGNER, S. 1990, A nalphabétisme de minorité et A Iphabétisation d'affirmation nationale à propos de I'O ntario français Alpha communautaire ches les Franco- $O$ ntariens, 9, Toronto, $M$ inistère de l'éducation.

WALLEN , J. (1992). «Providing C ulturally A ppropriate M ental H ealth Services for M inorities», T he Journal of $\mathrm{M}$ ental $\mathrm{H}$ ealth A dministration, Vol. 19, no 3, 288-295.

W O O LFSO N , P. (1975) «Traditional French-C anadian Family Life Patterns and T heir Implications for Social Services in Vermont », A F ranco-A merican 0 verview (Vol. 2) Cambridge, N ational Assessment and D issemination C enter for Bilingual/Bicultural Education. 
WO O DWAR D,A.M . ,A. D. DW IN ELL and B.S.AR O N S (1992). «Barriers to M ental H ealth C are for $\mathrm{H}$ ispanic Americans: A Literature R eview and Discussion », The Journal of $M$ ental $H$ ealth Administration, Vol. 19, no 3, 224-236.

\section{Notes}

1. Nous aimerions remercier les membres du comité dont j'assumais la présidence et, tout particulièrement, $M$ adame Anne-M arie Couffin, coordonnatrice des Services en français du Conseil régional de santé deToronto, le Dr. G ary Freeman, Directeur du C hild D evelopment \& Counseling Services de N orth York G eneral H ospital, M adame Julie Gaudet, N urse M anager, Psychiatric \& A dolescent M edicine du H ospital for Sick Children, M adame Elisabeth Lin et Isobel Barnes de I'Institut Clarke de psychiatrie, M adame Mardy Sounders du North York G eneral $\mathrm{H}$ ospital et $\mathrm{M}$ adameA nne $\mathrm{M}$ olgat, recherchiste et rédactrice qui ont tous participé à la réalisation du rapport. Les commentaires et réflexions exprimés dans cet article sont mon unique responsabilité. Enfin, nous voulons remercier chaleureusement les vingt- deux personnes qui ont accepté de partager leurs expériences personnelles avec nous afin de nous aider à mieux comprendre la situation des francophones en 0 ntario qui ont de graves problèmes de santé mentale à l'aube de l'an 2000.

2. Depuis l'avènement de la M égacitée, en 1998 , la municipalité métropolitaine de Toronto, comprenant Toronto, Scarborough, East York, N orth York, York et Etobicoke, est maintenant connue sous le nom de Toronto. La région du Grand Toronto, regroupe en plus deToronto, les divisions de $\mathrm{D}$ urham, York, Peel et $\mathrm{H}$ alton.

3. La grande priorité de la réforme était, en effet, de répondre aux beso ins des personnes et familles qui sont aux prises avec des troubles de santé mentale sévères et persistants, c'est-à- dire où la personne est 1) munie d'un diagnostic tel que schizo phrénie, troubles affectifs graves, syndrome cérébraux organiques, psychoses et autres; 2) dont l'invalidité est telle qu'elle l'empêche de fonctionner normalement et, 3) dont la durée se rapporte à la nature chronique de la maladie.

4. Le document identifiait certains groupes devant recevoir une attention spéciale au niveau de la planification des services: les femmes, les enfants, les adolescents, lesfrancophones, les autochtones, les membres de groupes ethnoculturels et les gens à diagnostic mixte (1993: 31)

5. N ous devons, en surcroît, noter la grande diversité ethnoculturelle de cette minorité francophone qui n'est pas mise en évidence dans ce portrait. Un francophone sur cinq àToronto appartient à une minorité visible (20\%) et presque un francophone sur trois est un immigrant (30,3\%) dont $47,5 \%$ viennent de pays Européens et les autres sont en provenance d'A frique, d'A sie, du M oyen0 rient, de l'A ustralie et des Iles M aurices.

6. La minorité francophone comprend les personnes qui ont le français comme première langue officielle parlée et la moitié des personnes qui ont le français et l'anglais. La première langue officielle parlée est une variable constituée pour les fins d'application de la Loi sur les langues officielles. Elle tient compte, premièrement, de la connaissance des langues officielles, deuxièmement, de la langue maternelle et, troisièmement de la langue parlée à la maison (Guindon, $R$ (1994). «D ossier statistique sur la francophonie ne la région métropolitaine de Toronto et de ses environs ». La Société d'études et de conseil AC O R D incorporée: 10). 
7. Les enquêteurs ont utilisé une version du Composite International Diagnostic Interview (CIDI) de l'U niversité du M ichigan développée par I'O rganisation M ondiale de la Santé qui peut s'administrer par questionnaire. Les entrevues sont structurées de façon à pouvoir identifier et classifier les désordres mentaux selon les définitions et critères contenus dans le D SM - IIIR publié par l'Association américaine de psychiatrie. Les résultats offrent une approximation considérée comme raisonnable d'un diagnostic qui serait posé par un professionnel lors d'une évaluation clinique. Le questionnaire incluait aussi le niveau d'invalidité encourue et de la perception qu'une personne se fait de sa propre santé mentale.

8. L'échantillon étant trop petit pour se concentrer uniquement surToronto.

9. C ette apparente absence de cohérence dans les résultats obtenus, reflète la complexité de la situation et doit être examinée entre autres en relation avec la présence ou non d'un système d'assurance maladie.

10. Les réponses offertes par la population francophone au Supplément sur la Santé M entale n'avaient jamais été examinées. Pour plus d'informations sur l'enquête, consultez le guide de l'usager de I'E nquête Santé de l'O ntario, M inistère de la Santé, 1992.

11. Les Francophones sont définis ici en termes de la langue parlée à la maison et/ ou la présence d'une identité francophone.

12. Toujours posés à l'aide du CID I

13. Les tableaux et résultats complets de cette étude peuvent être consultés dans le rapport: T he $\mathrm{N}$ eeds of $\mathrm{F}$ rancophones with Severe $\mathrm{M}$ ental $\mathrm{H}$ ealth P roblems in M etropolitan Toronto: «I only have the Words in French ». Les différences présentées ici ne sont pas énormes, mais assez pour suggérer une tendance. (Boudreau 1996).

14. Patrice D esbiens, cité dans François Paré, 1994 : 131- 132.

15. Le questionnaire semi-structuré a été soumis à un groupe-pilote composé de six personnes ayant des problèmes de santé mentale et dont la langue maternelle était autre que l'anglais ou le français. Le comité d'éthique de l'H ôpital Général de N orth York a exigé qu'un psychologue soit toujours disponible au moment où se faisaient les entrevues. Aucune personne en état de crise ou en phase aiguë de sa maladie n'a été interviewée. Les entrevues ont duré entre une et trois heures et se sont déroulées sans incidents. La moitié se sont faites par téléphone et l'autre en personne. Seule la personne faisant les entrevues connaissait l'identité des répondants et tout indice a été détruit après l'entrevue afin de préserver la confidentialité.

16. Certains adolescents contactés par leur médecin acceptent d'abord de participer. Pourtant, ils informent la personne qui feral'entrevue qu'ilsn'ont vraiment pas de problème de santé mentale. 